\title{
Numerical Analysis of Elongation Behavior of Melt Flow in Contraction Channels with Cylindrical Barriers
}

\author{
Alfeus SunARSO, Takehiro YAMAmOTO, and Noriyasu MORI \\ Department of Mechanophysics Engineering, Osaka University, \\ 2-1 Yamadaoka, Suita, Osaka 565-0871, Japan \\ (Received : November 15, 2004)
}

\begin{abstract}
The flow of polymer melt in contraction channels with cylindrical barriers was numerically simulated. A commercial LDPE melt was considered, and the rheological property of the melt was represented using the Extended Pom-Pom model (XPP). The simulation was performed with various barrier configurations, and the effects of the barriers were investigated extensively in relation to the efforts to clarify the phenomenon of melt fracture mitigation by the addition of filter or porous medium. It can be observed that the addition of barriers modifies significantly the flow fields in the region near the contraction. Investigation of the elongation stress shows that the addition of barriers increases the stress in the region near the contraction, but decreases the elongation rate and elongation stress in the region at the upstream of the contraction and induces the undershoots of elongation rate and elongation stress in the region at the downstream of the contraction. With the increase in Weissenberg numbers, these effects are more significant for the channels with two barriers as compared to that with single barrier. The reduction of elongation rate and elongation stress should play an important role in the mitigation of the upstream instability, which in turn mitigates the gross melt fracture.
\end{abstract}

Key Words: Numerical simulation / Pom-Pom model / Contraction channels / Barrier effects / Elongation behaviors

\section{INTRODUCTION}

In polymer processing, for example extrusion process, filter or porous medium is commonly used to separate the contaminating particles from the products. The addition of filter or porous medium increases the power required to perform the extrusion, but in the other side it can improve the processabilty of polymer melts. Actually, extrusion of polymer melts at high extrusion speed encounters various extrusion defects such as sharkskin, stick-slip and gross melt fracture, as clearly identified in the review by Petrie and Denn. ${ }^{1)}$ Considering the effect of filter or porous medium, it is interesting to note that the addition of filter or porous medium at the entrance of contraction can mitigate the gross melt fracture defect ${ }^{2-4}$ ), thus increases the possible speed for the process. However the mechanism of the phenomenon has been poorly understood yet, and further investigation is required.

The literature suggests that the origin of gross melt fracture is different from the origin of surface defects (sharkskin or stick-slip). Many researchers agree that the surface defects are initiated by slip in the die exit region, while the gross melt fracture is initiated by the flow instability in the region upstream the contraction ${ }^{5-9}$ ), but the accurate mechanism governing the instability is not yet completely clear. Regarding to the mitigation of gross melt fracture by the addition of filter or porous medium, several mechanisms were proposed, such as temporary change in rheological property by the disentanglement of polymer molecules ${ }^{2)}$ and reduction of stress overshoot by pre-shearing. ${ }^{3)}$ Recently, Goutille and Guillet ${ }^{4)}$ suggested that the modification of stress field by the modification of channel geometry should play an important role in the mitigation of the upstream instability, which in turn mitigates the gross melt fracture defect. However, the data that show how the stress field changed were not available yet. In the present work, we performed numerical simulation to investigate the effects of barriers on the stress fields as well as velocity field and the microstructure fields of the fluid.

We considered the flow in 4:1 contraction channels with various barrier configurations. The representative geometry of the flow channels is shown in Fig. 1, and the barrier configurations are shown in Table I. The melt of commercial LDPE called Stamylan (DSM, LD 2008 XC43) was considered. Rheological property of this melt represent the property of LDPE melts in general, which is characterized by extreme strain hardening at low strain rates and strain thinning at high strain rates, while the shear rheology is very shear 
Table I Channels and barrier configurations.

\begin{tabular}{llrr}
\hline \hline Channel & $\mathrm{N}$ & $\mathrm{X}$ & $\mathrm{Y}$ \\
\hline \hline $\mathrm{C} 000$ & 0 & - & - \\
\hline $\mathrm{C} 100$ & 1 & $-0.1 H$ & 0 \\
\hline $\mathrm{C} 110$ & 1 & 0 & 0 \\
\hline $\mathrm{C} 120$ & 1 & $0.1 H$ & 0 \\
\hline $\mathrm{C} 200$ & 2 & $-0.1 H$ & $\pm 0.3 H$ \\
\hline $\mathrm{C} 210$ & 2 & 0 & $\pm 0.3 H$ \\
\hline $\mathrm{N}:$ number of barriers & & \\
$\mathrm{X}:$ location of barrier center in $x$ direction \\
$\mathrm{Y}:$ location of barrier center in $y$ direction \\
\hline \hline
\end{tabular}

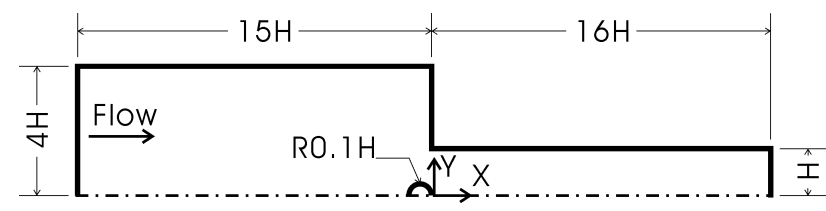

Fig. 1. Representative geometry of flow channels.

thinning. To correctly represent the rheological property of the melt, we used a molecular base constitutive equation, that is the multi-mode Extended Pom-Pom (XPP) model, which shows a better predictive capability as compared to the conventional constitutive equations such as the Phan-Thien Tanner (PTT) and the Giesekus model. ${ }^{10)}$ Another advantage of the XPP model is that the information of fluid microstructure such as molecular orientation and molecular stretch can be obtained.

\section{GOVERNING EQUATIONS}

In the present work, we consider the flow of incompressible isothermal polymer melts, which is governed by the following conservation equations:

$$
\begin{aligned}
& \rho\left(\frac{\partial \boldsymbol{u}}{\partial t}+\boldsymbol{u} \cdot \nabla \boldsymbol{u}\right)=-\nabla p+\nabla\left(\boldsymbol{\tau}_{p}+2 \eta \boldsymbol{D}\right) \\
& \nabla \cdot \boldsymbol{u}=0
\end{aligned}
$$

Here, $\rho$ is the fluid density, $\boldsymbol{u}$ is the velocity vector, $p$ is the pressure, $\tau_{p}$ is the polymeric stress tensor, $\eta$ is the Newtonian viscosity, and $\boldsymbol{D}$ is the rate of deformation tensor. Because polymer melts usually have a high value of viscosity, the effect of gravity on the flow can be neglected.

The polymeric stress $\tau_{p}$ is computed using the multi-mode XPP model. In this model, the stress is computed from the evolution equations of molecular orientation tensor $\boldsymbol{S}$ and molecular backbone stretch $\Lambda$. Considering the two dimensional case, in which the second normal stress difference can be neglected and the second normal stress factor $\alpha$ can be set to zero, the constitutive equations are as follows:

$$
\begin{aligned}
& \boldsymbol{\tau}_{p}=G\left(3 \Lambda^{2} \boldsymbol{S}-\boldsymbol{I}\right) \\
& \frac{D \Lambda}{D t}=\Lambda(\boldsymbol{\kappa}: \boldsymbol{S})-\frac{1}{\lambda_{s}}(\Lambda-1) e^{v^{*}(\Lambda-1)} \\
& \frac{D \boldsymbol{S}}{D t}=\boldsymbol{\kappa} \cdot \boldsymbol{S}+\boldsymbol{S} \cdot \boldsymbol{\kappa}^{T}-2(\boldsymbol{D}: \boldsymbol{S}) \boldsymbol{S}-\frac{1}{\lambda_{b} \Lambda^{2}}\left(\boldsymbol{S}-\frac{\boldsymbol{I}}{3}\right)
\end{aligned}
$$

where $G$ is a plateau modulus, $\boldsymbol{k}$ is the velocity gradient tensor $\left(k_{i j}=\partial u_{i} / \partial x_{j}\right), \lambda_{s}$ is the relaxation time of backbone stretch, $\lambda_{b}$ is the relaxation time of backbone orientation, and $\boldsymbol{I}$ is the identity tensor.

\section{NUMERICAL METHODS}

The governing equations (1)-(5) are solved using a decoupled approach. The velocity and pressure fields are computed simultaneously by solving the conservation equations (1) and (2) using a standard finite element method, while the polymeric stress is obtained by solving the set of constitutive equations (3)-(5) using the backward-tracking Lagrangian particle method (BLPM). ${ }^{11)}$ The stability of computation is improved by including the discrete elastic stress splitting (DEVSS) method. ${ }^{10,11)}$ To reduce the computational time, the modes with short relaxation time are treated as Newtonian fluids. ${ }^{12)}$ For the approximation spaces, we used biquadratic continuous, bilinear continuous and bilinear discontinuous representations for velocity, pressure and polymeric stress, respectively.

\section{SIMULATION CONDITION}

\subsection{Material Characterization}

We considered a commercial LDPE melt that is known as Stamylan. The rheological property of the melt is represented using four modes XPP model, and the fitting parameters are shown in Table II. These are the same parameters as proposed by Verbeeten ${ }^{10)}$, except that the second normal stress difference factors $\alpha$ are zero. Furthermore, the first mode is treated as a Newtonian fluid. ${ }^{12)}$ In this case, the Newtonian viscosity $\eta_{s}$ is 
Table II Fitting parameters for the XPP model.

\begin{tabular}{cccc}
\hline \hline Mode & $G[\mathrm{~Pa}]$ & $\lambda_{s}[\mathrm{~s}]$ & $\lambda_{b}[\mathrm{~s}]$ \\
\hline \hline 1 & $7.2006 \times 10^{4}$ & $5.5637 \times 10^{-4}$ & $3.8946 \times 10^{-3}$ \\
\hline 2 & $1.5770 \times 10^{4}$ & $1.0278 \times 10^{-2}$ & $5.1390 \times 10^{-2}$ \\
\hline 3 & $3.3340 \times 10^{3}$ & $1.6783 \times 10^{-1}$ & $5.0349 \times 10^{-1}$ \\
\hline 4 & $3.0080 \times 10^{2}$ & $4.1737 \times 10^{0}$ & $4.5911 \times 10^{0}$ \\
\hline \hline
\end{tabular}

$208 \mathrm{~Pa} \cdot \mathrm{s}$ and zero shear viscosity of polymer $\eta_{p}$ is $3870 \mathrm{~Pa} \cdot \mathrm{s}$, and viscosity averaged relaxation time $\bar{\lambda}$ is $1.7415 \mathrm{~s}$.

\subsection{Weissenberg Numbers}

The simulation was performed under various flow rates, which correspond to various Weissenberg numbers, $W i$ which are defined as follows:

$$
W i=\frac{\bar{\lambda} \bar{u}}{H}
$$

Here, $\bar{u}$ is the average velocity and $H$ is the half width of the channel at the exit. In the simulation, Weissenberg numbers of $0.3,1.0,5.0$ and 10.0 are considered.

\section{RESULTS AND DISCUSSION}

\subsection{Overview}

In our previous work ${ }^{13,14)}$, we have shown that the addition of barriers significantly modifies the flow fields near the contraction. This can be observed in the modification of velocity fields, which expressed in the term of streamline, as shown in Fig. 2. The modification of velocity field in the region upstream of the contraction resulted in the modification of vortex size, which depends on both $W i$ and barrier configurations. Further investigation on the stress fields showed that the addition of barrier increases significantly the first normal stress difference $N_{1}=\tau_{x x}-\tau_{y y}$ and shear stress $\tau_{x y}$ in the region near the contraction, but reduces the stress in the region at the upstream of the contraction.

As we noted in the introduction, the flow instability in the entrance region is considered as a trigger of gross melt fracture. In this region, significant extensional stresses prevail, and a critical extensional stress appears to be the criterion for the onset of gross melt fracture. ${ }^{\text {) }}$ Furthermore, Sentmanat and Hatzikiriakos ${ }^{15)}$ have suggested that the suppression of elongation stress growth by the addition of a small amount of boron nitride into a polyethylene is responsible in the mitigation of gross melt fracture. Considering these results, we performed further analysis of the modification of elongation behaviors by the addition of barrier.

To obtain the elongation properties, for example, elongation stress $\sigma_{e l}$, the stress tensor $\boldsymbol{\sigma}$ is projected along the streamline by constructing a unit tangential vector $\boldsymbol{t}$ based on the segment of the streamline, and then performing the following operation:

$$
\sigma_{e l}=\boldsymbol{t} \cdot \boldsymbol{\sigma} \cdot \boldsymbol{t}
$$

In the same way, the elongation rate can be obtained by projecting the rate of deformation tensor $\boldsymbol{D}$ to the streamline.

\subsection{Modification of Elongation Behaviors}

The modification of elongation rate and elongation stress fields by the addition of barriers is shown in Fig. 3. As shown in Fig. 2, the addition of barrier promotes the acceleration of fluid before entering the contraction, but promotes the deceleration of fluid in the region just at the downstream of the contraction. As the consequence, in the region just upstream of the contraction the elongation rates increase, but in the region just downstream of the contraction, the elongation rates decrease to negative values, as shown in Fig. 3a. Consequently, the change in the elongation rate fields modifies the elongation stress fields, as shown in Fig. 3b. It can be observed that the addition of barrier increases the elongation stress in contraction region, but decreases the stress in the region at a distance at the upstream and the downstream of the contraction.

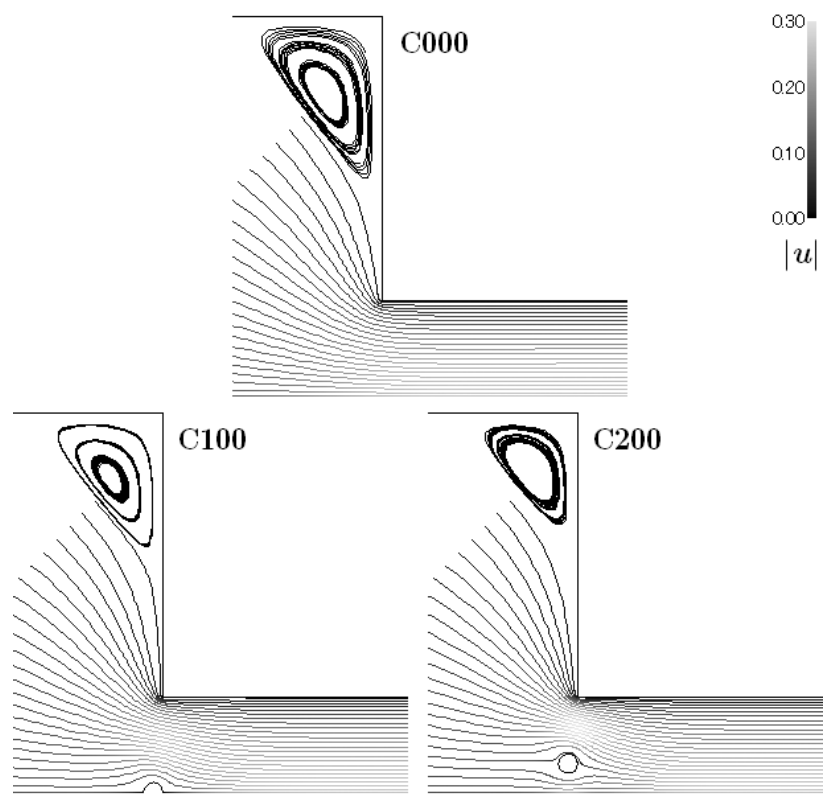

Fig. 2. Modification of streamline patterns at $W i=0.3$. 
Further analysis is performed by observing the elongation rate and elongation stress distributions in the horizontal cross section at $y=0.6 H$. In Fig. 4, we can observe the overshoots in the elongation rate at the upstream of the contraction for all channels, while in the region at the downstream of the contraction, undershoots are clearly observed only for the channels with barrier. The overshoots and undershoots in the
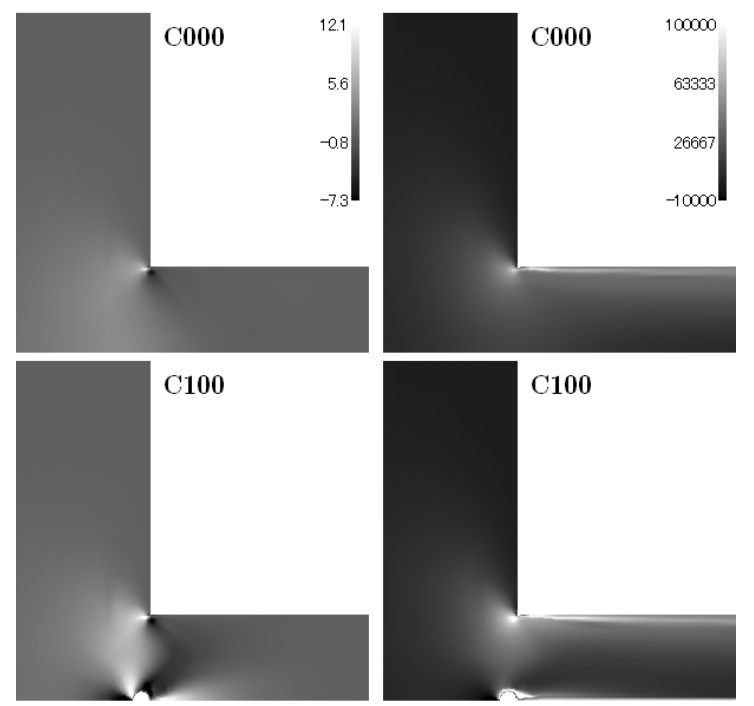

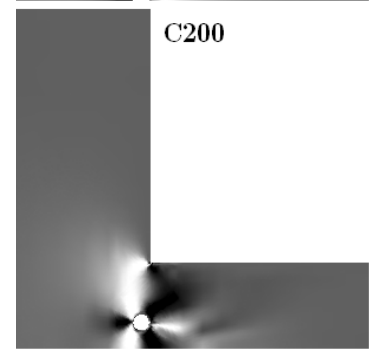

(a) elongation rate

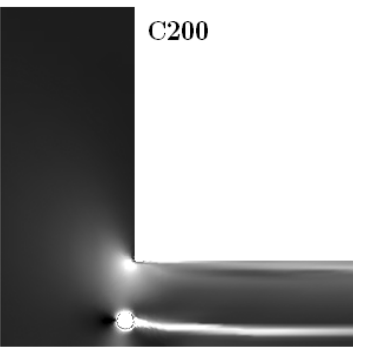

(b) elongation stress
Fig. 3. Modification of patterns of elongation rate (left) and elongation stress (right) at $W i=10$.

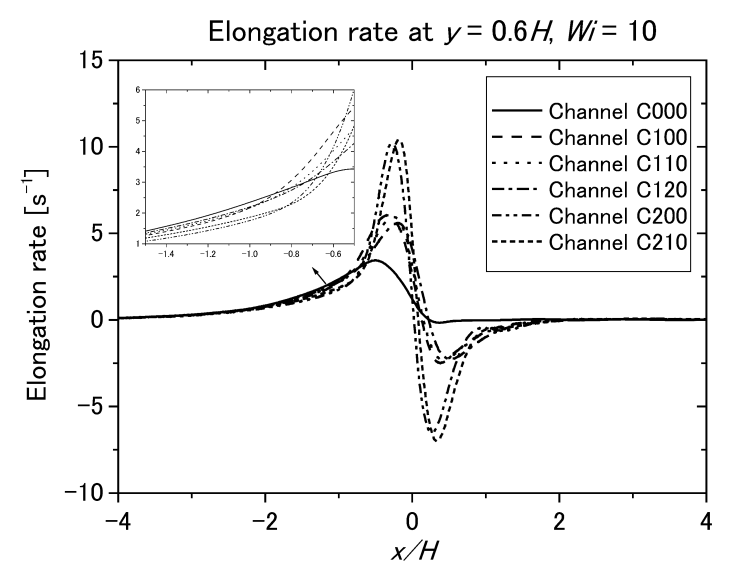

Fig. 4. Distribution of elongation rate at cross section of $y=0.6 \mathrm{H}$ at $W i=10$. elongation rate are related to the acceleration and deceleration of fluid respectively. As the barrier promotes the acceleration and deceleration, it can be seen that the addition of barrier increases the magnitude of the overshoots and undershoots. Furthermore, the addition of barrier moves the location where the stress start to increases to the downstream, and thus in the region at a distance away from the upstream of the contraction, the elongation rate is lower for the channels with barrier as compared to that without barrier. Even though the absolute values of elongation rate vary with $W i$, the distribution curves show the similar pattern.

The change in elongation rate distributions directly affects the distributions of elongation stress, as shown in Fig. 5. At $W i=0.3$, as the consequence of overshoots and undershoots in the elongation rate, the stress overshoots and undershoots can be observed for all channels. The amplitude of the overshoots and undershoots increases as the number of barrier increases. At $W i=10$, stress undershoot disappears for channel without
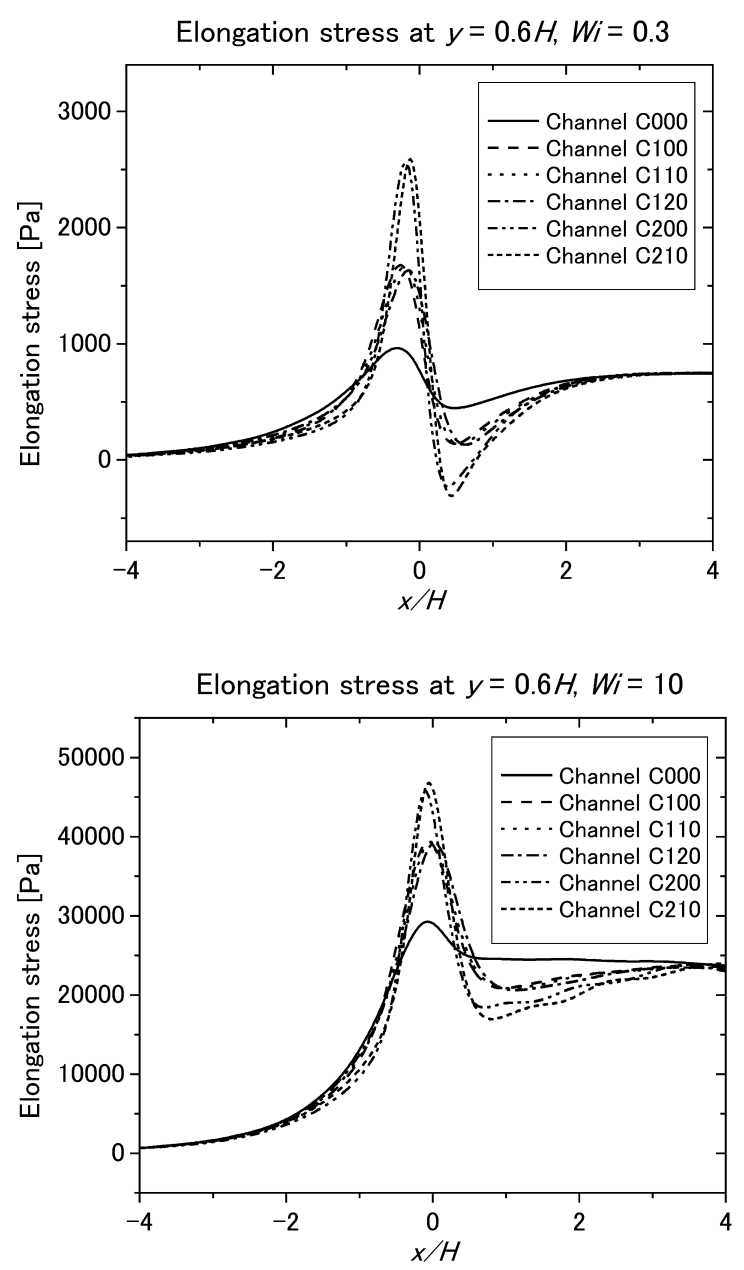

Fig. 5. Distribution of elongation stress at cross section of $y=0.6 H$, $W i=0.3$ (upper), $W i=10$ (lower). 
barrier, but it still appears for channels with barrier. The preservation of stress undershoots in the case of channels with barrier is related to the existence of region with negative elongation rate, which should reduce the high stress convected by fluid from the upstream. Furthermore, as the barrier decreases the elongation rate in the region at a distance away from the upstream of the contraction, the reduction of elongation stresses can be observed in that region both at $W i=0.3$ and $W i=10$.

To investigate the effect of barrier on the reduction of stress in more detail, we observed the elongation stress distribution in the upstream region, that is at vertical cross section of $x=-H$, as shown in Fig. 6. We can observe that the addition of barrier decreases the elongation stress in the region between the centerline $(y=0)$ and the wall of channel exit $(y=H)$. With the increase in $W i$, the reduction becomes more significant as number of barrier increases. This can be confirmed by observing the elongation stress at point of $x=-H$ and $y=0$ at various $W i$, as shown in Fig. 7. It can be seen that as the number of barrier increases, the growth of elongation stress with the increase in $W i$ becomes smaller.

\subsection{Barrier Effects and Upstream Instability}

As noted previously, it is agreed that the gross melt fracture is related to the flow instability in the region upstream of the contraction. However, the mechanism governing the instability is not yet completely clear and needs further investigation. In the case of polymer solution, when the upstream instability occurred, a high velocity fluid jet from the corner of contraction enters the exit channel ${ }^{16,17)}$ that causes the buckling like flow structure, in which the flow crosses the centerline and losses its symmetry in the upstream region. In the case of polymer melt, it is also reasonable to consider that the upstream instability is triggered by the similar mechanism. The work of Legrand and Piau ${ }^{18)}$ confirmed that the upstream instability begins by small velocity fluctuations along streamlines in the lateral zone of contraction, i.e., near the corners. Birefringence measurements show the oscillation of stress in the region near the corner. This oscillation propagates to the downstream region and its amplitudes increase as the flow rate increases.

Considering our simulation results, we suggest that the reduction of elongation rate and elongation stress in the upstream region and the existence of undershoots in elongation rate and elongation stress in the downstream region should play an important role in the mitigation of upstream instability, which in turn mitigates the gross melt fracture. The region with negative elongation rate (or low elongation stress) at the downstream should serve as a damper that prevents the disturbance to propagate to downstream, while relatively low elongation rate in the upstream region prevents the amplification of disturbance in the upstream flow. Therefore, even though the addition of barrier increases the stress in the contraction region that would increase the occurrence of disturbance in this region, the existence of region with relatively low elongation rate at the upstream and downstream localizes the effects of the disturbance, thus prevents the occurrence of bulk instability. The experimental result ${ }^{4)}$ shows that at the onset of gross melt fracture, although small pulsation is still observed in the upstream of the contraction, bulk oscillation of flow in the upstream is clearly diminished and the melt fracture defect is reduced by the addition of filter.

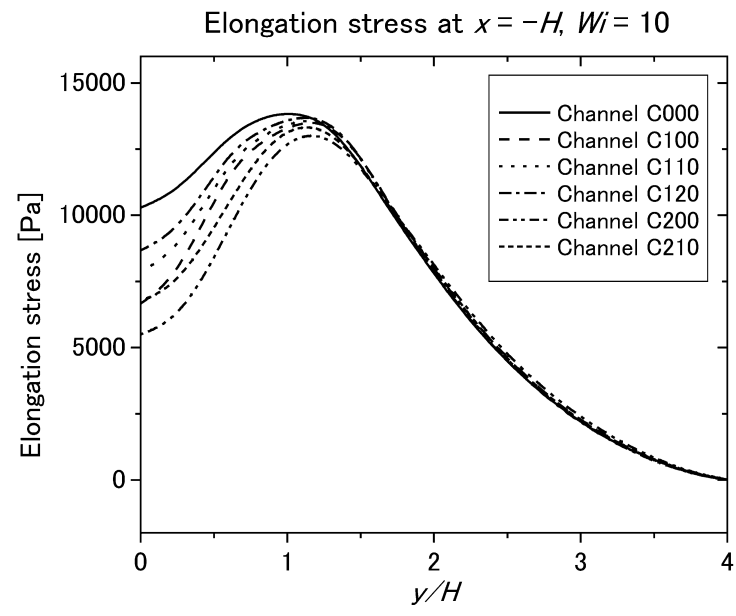

Fig. 6. Distribution of elongation stress at cross section of $x=-H$ at $W i=10$.

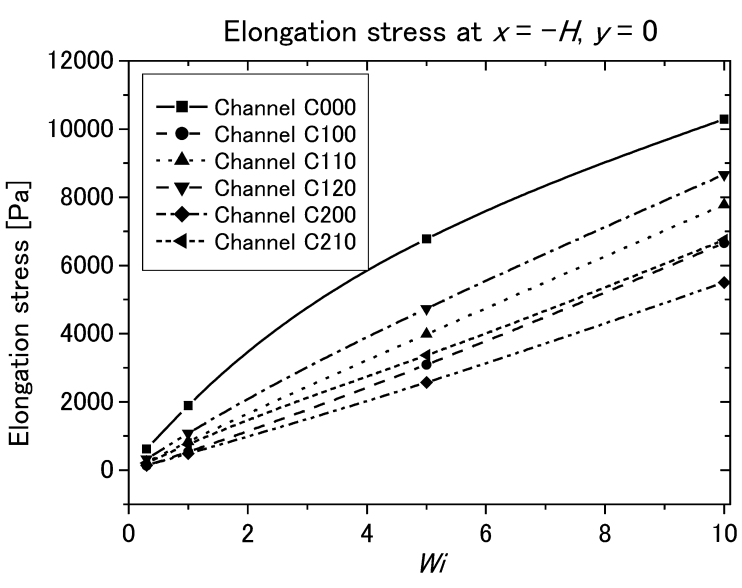

Fig. 7. Elongation stress at point of $x=-H, y=0$. 


\subsection{Modification of Fluid Micro Structure}

Besides the modification in velocity and stress fields, the addition of barriers also affects the molecular configurations, which should relate to the mechanical and optical properties of the product. At low Wi, the modification of molecular configuration in the region far downstream of the barrier is not significant, but it becomes significant as $W i$ increases. Figure 8 shows the distribution of orientation order parameter $S_{x x}$ of mode with the longest relaxation time at vertical cross section of $x=10 H$ at $W i=10$. Without barrier, the molecules are highly oriented to the flow direction in the region near the wall. In the region near the centerline, the molecules are less oriented. The addition of barrier introduces the region with high elongation rate, which extends to the downstream and causes the molecules to be more oriented to the flow direction in this region.

\section{CONCLUDING REMARKS}

Numerical simulation has been performed to investigate the effects of cylindrical barriers on the flow of polymer melt in the contraction channels. It can be observed that the addition of the barrier increases significantly the elongation rate and

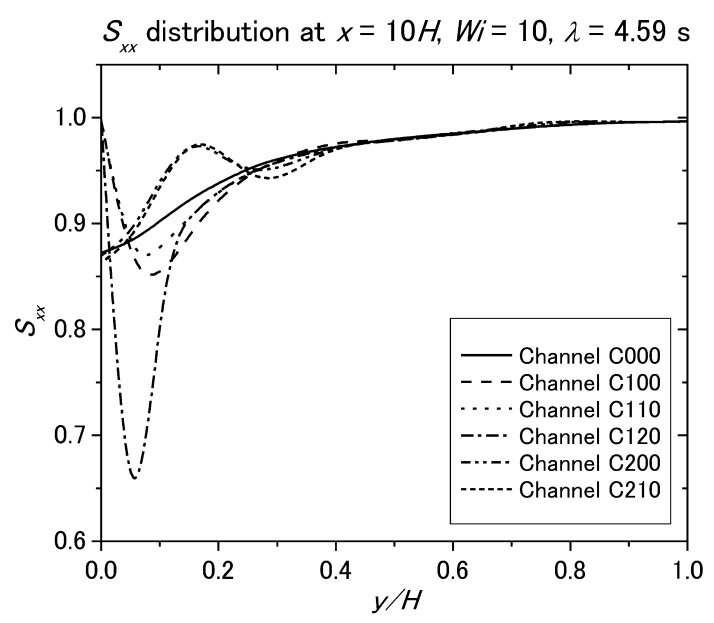

Fig. 8. Distribution of orientation parameter $S x x$ at cross section of $x=10 \mathrm{H}$ at $\mathrm{Wi}=10$. elongation stress in the contraction region, but introduces the region with relatively low elongation rate and elongation stress at the upstream and region with negative elongation rate and low elongation stress at the downstream of contraction. It is suggested that these regions should play an important role in the localization of disturbance that may occur in the contraction region, which in turn mitigate the upstream instability that is considered as the trigger of the gross melt fracture.

\section{REFERENCES}

1) Petrie CJS, Denn MM, AIChE J, 22, 209 (1976).

2) Done DS, Baird DG, Average AE, Chem Eng Commun, 21, 293 (1983).

3) Piau JM, Nigen S, El Kissi N, J Non-Newtonian Fluid Mech, 91, 37 (2000).

4) Goutille Y, Guillet J, J Non-Newtonian Fluid Mech, 102, 19 (2002).

5) White JL, J Appl Polym Sci, 8, 2339 (1964).

6) Cogswell FN, Polym Eng Sci, 12, 64 (1972).

7) Cogswell FN, J Non-Newtonian Fluid Mech, 2, 33 (1977).

8) Tordella JP, Trans Soc Rheol, 1, 203 (1957).

9) Piau JM, El Kissi N, Tremblay B, J Non-Newtonian Fluid Mech, 34, 145 (1990).

10) Verbeeten WMH, Peters GWM, Baaijens FPT, $J$ NonNewtonian Fluid Mech, 108, 301 (2002).

11) Wapperom P, Keunings R, J Non-Newtonian Fluid Mech, 91, 273 (2000).

12) Lee K, Mackley MR, McLeish TCB, Nicholson TM, Harlen OG, J Rheol, 56, 1261 (2001).

13) Sunarso A, Yamamoto $T$, Mori N, Proc XIVth Int Congr on Rheology, NF27, 2004.

14) Sunarso A, Yamamoto T, Mori N, to appear in Proc 82nd JSME Conf on Fluid Eng, 2004.

15) Sentmanat M, Hatzikiriakos SG, Rheol Acta, Online First, 20 March, 2004.

16) Chiba K, Nakamura K, J Non-Newtonian Fluid Mech, 73, 67 (1997).

17) Rothstein JP, McKinley GH, J Non-Newtonian Fluid Mech, 98, 33 (2001).

18) Legrand F, Piau JM, J Non-Newtonian Fluid Mech, 77, 123 (1998). 